\section{Corporate Members}

$$
\text { PATRONS }
$$

Alberta Geological Survey

Anglo American Exploration (Canada) Ltd.

Memorial University of Newfoundland

Newfoundland and Labrador Department of Natural Resources

Northwest Territories Geoscience Office

Energy, Mines and Resources - Government of Yukon

\section{SPONSORS}

Royal Tyrrell Museum of Palaeontology

Saskatchewan Geological Survey

\section{SUPPORTERS}

Activation Laboratories Ltd.

Elemental Controls Ltd.

Franklin Geosciences Ltd.

Geosciences Laboratory

IBK Capital Corp.

Isomass Scientific Ltd.

Living Sky Geophysics Inc.

$$
\begin{gathered}
\text { UNIVERSITIES } \\
\text { Acadia University }
\end{gathered}
$$

INRS Eau Terre Environnement Centre

Laurentian University

McGill University

Université Genève

Université du Québec à Montréal

University of New Brunswick

University of Toronto

University of Waterloo
GEOLOGICAL

ASSOCIATION OF

CANADA

(2013-2014)

\section{OFFICERS}

President

Richard Wardle

Vice-President

Brian Pratt

Past President

Peter Bobrowsky

Secretary-Treasurer

Toby Rivers

\section{COUNCILLORS}

Shannon Acton

Kathryn Bethune

Peter Bobrowsky

David Corrigan

John Hanchar

Lori Kennedy

Ray Lett

Francine McCarthy

Brad McKinley

Brendan Murphy

Brian Pratt

Toby Rivers

Martin Ross

Stephen Rowins

Victoria Sterritt

Mike Villeneuve

Richard Wardle

Chris White

\section{STANDING COMMITTEES}

Communications: Mike Villeneuve

Finance: Francine McCarthy

Publications: Chris White

Science Program: Martin Ross 